\title{
Intestinal pseudo-obstruction
}

\author{
M A Kamm
}

Intestinal pseudo-obstruction refers to a syndrome in which the clinical picture resembles that of bowel obstruction, with failure of intestinal motility associated with pain and intestinal distension, but absence of a mechanical obstructing lesion.

The situation arises acutely in some patients admitted to hospital with non-gastrointestinal disorders (Ogilvie's syndrome), such as myocardial infarction, pneumonia, or fractured hip. Typically there is acute colonic dilatation, with pain and abdominal distension. The condition is thought to be due to an imbalance of extrinsic autonomic nerves innervating the gut, with excessive sympathetic inhibitory activity or diminished prokinetic cholinergic drive. The condition can be managed by colonic decompression, either colonoscopically or surgically. More recently, the acute use of an anticholinesterase such as neostigmine has been shown to cause rapid resumption of normal activity, adding weight to the aetiological theory of autonomic nerve dysfunction.

The chronic condition of intestinal pseudoobstruction relates to a clinical syndrome of persistent dysfunction over a prolonged period. The condition may be secondary to neuromuscular diseases such as scleroderma, bronchial carcinoma, or amyloidosis. Alternatively, it may be primary, with the disease usually selectively affecting either intestinal smooth muscle or enteric nerve plexuses. ${ }^{1}$ These myopathies or neuropathies can be either degenerative or inflammatory.

These chronic conditions might be thought to be a useful model regarding the selective role played by enteric nerves or enteric muscle. In practice however, the myopathies and neuropathies have similar clinical manifestations, with pain, gut dilatation, and diminished coordinated gut motor activity. ${ }^{2}$

A number of important aetiological factors have emerged over recent years. The use of immunohistochemical techniques has allowed the identification of biochemical abnormalities in some patients, whereas previously only structural or morphological diagnosis was possible. An abnormality of enteric smooth muscle alpha actin has been identified in a patient with normal histology. ${ }^{3}$ Ebstein-Barr virus has been identified on polymerase chain reaction and in situ hybridisation, localised to the myenteric plexus, in a patient with chronic pseudoobstruction due to inflammatory plexitis. ${ }^{4}$

Therapy of these disorders has so far remained confined to symptomatic support, pain relief, nutritional support, and treatment of complications such as bacterial overgrowth. Specific drug therapies have proved disappointing. A randomised trial of cisapride failed to show a marked therapeutic effect. ${ }^{5}$

One limitation of drug studies in this condition has been failure to recognise that this is not a uniform clinical condition, but one comprising a range of aetiologies. It would therefore be more appropriate to test drug therapies using an $n=1$ trial design, employing multiple drug trials in one patient to determine response. In a small group of patients, erythromycin, the motilin analogue, appears to enhance gut function and avert episodes of ileus. In others, the 5-hydroxytryptamine-4 (5- $\left.\mathrm{HT}_{4}\right)$ agonists being developed may be helpful.

Although the histopathology of some patients suggest chronic end stage disease, the clinical picture often fluctuates with periods of relative good health with less symptoms, interspersed with episodes of complete ileus. This suggests that some unrecognised factors superimposed on background gut pathology contribute to episodes of clinical worsening. However, such fluctuation also offers the hope that specific (drug) therapies may still have a role, despite the pathology present.

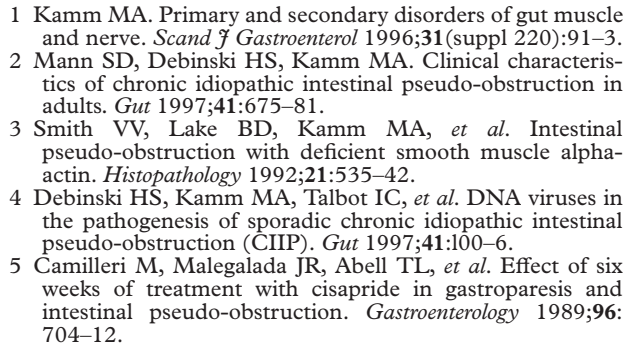
704-12.

Abbreviations used in this paper: 5- $\mathrm{HT}$, 5-hydroxytryptamine. 